\title{
Weighted Centroid Localization Algorithm Based on Normal Distribution in Wireless Sensor Networks
}

\author{
Shaoguo XIE \\ School of Computers and Information \\ Anqing Normal University \\ Anqing, Anhui Province, China \\ e-mail: xieshaoguo@126.com
}

\begin{abstract}
Localization algorithms continue to be a challenging and important topic in wireless sensor networks (WSNs). To efficiently exploit the normal distribution for range-free localization, we propose two weighted centroid localization algorithms called Positioning Based on Normal Distribution and Positioning Based on the Network Size. The former uses the normal distribution to reduce weights errors. The latter takes a further step to control the variance in normal distribution to estimate the location. Simulation results show that the proposed weighted centroid algorithms outperform WCL and AMWCL-RSSI (anchor_optimized modified weighted centroid localization algorithm based on RSSI) in terms of the localization accuracy for WSNs with planned deployment of anchor nodes. Experimental results demonstrate that the proposed two algorithms have a small localization error.
\end{abstract}

Keywords-localization; normal distribution; wireless sensor networks; weighted centroid localization algorithm; range-free localization

\section{INTRODUCTION}

Wireless sensor networks (WSNs) are emerging as a key tool for many applications including environmental monitoring, emergency relief, battlefield surveillance, home automation, assisted living, and so on due to its reliability, miniaturization, lower cost, and ease of deployment. Location information is important for many WSN applications such as routing, and for achieving higher network security and energy-efficient node management [1].

This paper is motivated by one of our ongoing WSN projects, i.e., the monitoring of the bomb site, in which the location information of on-site personnel is critical. In our project, some anchor nodes are deployed in the bomb site, on-site personnel enter the interior of the bomb site with the mobile monitoring node. We know the environment on spot and the location of on-site personnel through anchor nodes and mobile monitoring nodes. Wireless signals of nodes are often tampered by the environmental factors, including the temperature and the gas mixture in the air. Actual experiences reveal that localization in the bomb site still remains very challenging, in spite of great efforts and results developed in the literature. Challenges come from various aspects. On one hand, nonuniform deployment of sensor nodes could affect the effectiveness of range-free localization. On the other hand, for range-based localization, the received signal strength (RSS) used for estimating distances is highly dynamic, irregular and asymmetric between pairs of nodes. To make it even worse, the complex terrain and obstacles in the bomb site can easily affect RSS-based measurements, thus incurring undesired but ubiquitous errors.

Range-free localization algorithms, such as centroid localization and weighted centroid localization [2], have already caused much attention due to their simplicity and robustness to changes in wireless transmission. These advantages make them suitable for the coarse localization of a blind node. Strictly speaking, the weighted centroid localization is not an entirely range-free technique because it needs extra information such as the RSS. Recently many proposed approaches on weighted centroid localization are focused more on error control and management. Weighted centroid localization (WCL) is firstly proposed in [3]. In [4], Hongyu et al. propose an anchor_optimized modified weighted centroid localization algorithm based on RSSI (AMWCL-RSSI). Other schemes have discussed inexact position problems [5], or analyzed the performance of WCL algorithm [6], or enhanced WCL's performance for specific scenarios $[7,8]$.

In this paper, we focus on improving the performance of WCL, and propose two WCL algorithms for WSNs with planned deployment of anchor nodes. We compare our proposed algorithms with some existing algorithms via simulations and experiments.

The rest of this paper is organized as follows. Section 2 presents the proposed positioning algorithms, followed by performance evaluation in Section 3. The conclusions and the future work are summarized in Section 4.

\section{PROPOSED Positioning AlgORITHMS}

\section{A. Wireless Channel Propagation Model}

In order to establish the propagation channel, we assume that the RSS obeys the log-distance shadowing path loss model in reference [6] because the log-distance shadowing path-loss model is more suitable for actual application environment. This propagation model is widely used in wireless link budget and is expressed by: 


$$
R S S(d)=P L 0-10 \beta \log _{10}\left(d / d_{0}\right)+N_{\alpha}
$$

Where $d$ is the real distance between two nodes, PLO is the received signal strength at reference distance $d_{0}\left(d_{0}=1 \mathrm{~m}\right), \beta$ is the path loss exponent, and $N_{\alpha}$ is a zero-mean Gaussian noise with standard deviation $\alpha$.

\section{B. Overview of Positioning System}

The aim of the RSS-based localization is to seek a mapping from the measurements to a physical location. One of the most important mapping functions is the probabilistic model finding the normalized weight $W_{i}[8,9]$, where $0<W_{i}<1$. Then, the location can be regarded as a regression problem as $(x, y)=\sum_{i=1}^{n} W_{i} I_{i}$ [9], where $n$ is the number of anchor nodes, $I_{i}$ represents the coordinate of the $i-t h$ anchor node and $(x, y)$ represents the estimated location. This paper adopts a novel approach to computing $W_{i}$.

\section{Positioning Based on Normal Distribution (PND)}

In this section, we will introduce PND for location estimation. Let us assume that each unknown node can hear $n$ anchor nodes. The variable $d_{i}$ represents the measured distance to anchor nodes $i$ from the unknown node. The coordinate $P=(x, y)$ is the estimated position of an unknown node.

In general, the closer an anchor node gets to the unknown node, the greater weight the anchor node has [3]. According to this relationship between the weight and the distance between an anchor node and an unknown node, we propose that the corresponding relation between the weight $w_{i}$ and the measured distance $d_{i}$ obeys the normal distribution $N\left(\mu, \sigma^{2}\right)$, where $\mu$ is the mathematical expectation and $\mu=0, \sigma^{2}$ is the variance. In this normal distribution, the weight $w_{i}$ and the measured $d_{i}$ have an inverse relationship.

$d_{i}$ is independent, so $\sigma^{2}=\frac{1}{n} \sum_{i=1}^{n} d_{i}^{2}, d_{i} \in(-3 \sigma,+3 \sigma)$. Therefore, $w_{i}=\frac{1}{\sqrt{2 \pi} \sigma} e^{-\frac{1}{2}\left(\frac{\left.d_{i}\right)^{2}}{\sigma}\right.}$.

After $w_{i}$ normalization, the normalized weight can be expressed as

$$
W_{i}=w_{i} /\left(\sum_{j=1}^{n} w_{j}\right)=e^{-\left(\frac{n d_{i}^{2}}{2 \sum_{k=1}^{n} d_{k}^{2}}\right)} /\left(\sum_{j=1}^{n} e^{-\left(\frac{n d_{j}^{2}}{2 \sum_{k=1}^{n} d_{k}^{2}}\right)}\right)
$$

From Eq. 2, the estimated position of an unknown node can be expressed as follows:

$$
(x, y)=\sum_{i=1}^{n} W_{i} I_{i}
$$

The normalized weight $W_{i}$ also obeys the relationship that the closer an anchor node gets to the unknown node, the greater weight the anchor node has [3]. In other words, $W_{i}$ and $d_{i}$ obeys the normal distribution $N\left(0, \sigma^{2}\right)$. From the above description, we also conclude that anchor nodes can be randomly deployed in a region.

The procedures for the PND algorithm are summarized as follows:

step1: compute the measured distance $d_{i}$ according to RSS model in Eq. 1.

step2: compute the variance $\sigma^{2}$ in normal distribution $N\left(0, \sigma^{2}\right), \sigma^{2}=\frac{1}{n} \sum_{i=1}^{n} d_{i}^{2}$, where $n$ is the number of arrived anchor nodes.

step3: compute $w_{i}=\frac{1}{\sqrt{2 \pi} \sigma} e^{-\frac{1}{2}\left(\frac{d_{i}}{\sigma}\right)^{2}}$ according to step1 and step2.

step4: compute the normalized weight $W_{i}$ in Eq. 2.

step5: compute the estimated position $(x, y)=\sum_{i=1}^{n} W_{i} I_{i}$.

\section{Positioning Based on the Network Size (PNS)}

In practice, $\sigma^{2}$ can affect the localization accuracy when $\sigma^{2}$ is too big or too small. If $\sigma^{2}$ is too big, weights will focus on anchor nodes which are far away from the unknown node. If $\sigma^{2}$ is too small, the weights will focus on anchor nodes closer to the unknown node. Hence, PNS is proposed to eliminate this effect.

If the network size meets the requirements of the standard deviation, we can obtain the standard deviation just by knowing the network size. In order to meet the requirements of the standard deviation, we must preset the network size, e.g., as shown in Fig. 1. In Fig. 1, four anchor nodes are respectively located at four corners, sensor nodes are randomly placed in the region, and we choose the network size (e.g. $L \times L\left(m^{2}\right)$ ) as $\sigma^{2}$. According to the geometric relationship, we can know that $0<d_{i}<\sqrt{2} L$ and the maximum $d_{i}=\frac{\sqrt{2}}{2} L$, so $L>\frac{d_{i}}{2}$. Therefore, $L>\frac{d_{i}}{3}$. We set $L^{2}=\sigma^{2}$, then $\sigma>\frac{d_{i}}{3}$, where $d_{i} \in(0,3 \sigma)$. Hence, $w_{i}=\frac{1}{\sqrt{2 \pi} \sigma} e^{-\frac{1}{2}\left(\frac{d_{i}}{\sigma}\right)^{2}}$. After $w_{i}$ normalization, the normalized weight can be expressed as

$$
W_{i}=w_{i} /\left(\sum_{j=1}^{n} w_{j}\right)=e^{-\frac{1}{2}\left(\frac{d_{i}}{\sigma}\right)^{2}} /\left(\sum_{j=1}^{n} e^{-\frac{1}{2}\left(\frac{d_{j}}{\sigma}\right)^{2}}\right)
$$


From Eq. 4, the estimated position of an unknown node can be expressed as follows:

$$
(x, y)=\sum_{i=1}^{n} W_{i} I_{i}
$$

The procedures for the PNS algorithm are summarized as follows:

step1: determine a $L \times L\left(\mathrm{~m}^{2}\right)$ region as the test region, such as shown in Fig.1. All anchor nodes are deployed at the edge of this region, sensor nodes are randomly placed in the region.

step2: compute the measured distance $d_{i}$ according to the RSS.

step3: compute the variance $\sigma^{2}$, where $\sigma^{2}$ is equal to the network size.

step4: compute $w_{i}=\frac{1}{\sqrt{2 \pi} \sigma} e^{-\frac{1}{2}\left(\frac{\left.d_{i}\right)^{2}}{\sigma}\right.}$ according to step1 and step2.

step5: compute the normalized weight $W_{i}$ in Eq. 4.

step6: compute the estimated position $(x, y)=\sum_{i=1}^{n} W_{i} I_{i}$.

\section{Performance Evaluation}

\section{A. Simulation}

In this section, we analyze the performance of the studied localization schemes through simulations, using Matlab as a simulation tool. We compare the two proposed weighted centroid localization algorithms with WCL [3] and AMWCL-RSSI [4]. In the simulation, we evaluate the effect of the size of the network has on the localization error. We simulated three network sizes: $10 \mathrm{~m} \times 10 \mathrm{~m}, 20 \mathrm{~m} \times 20 \mathrm{~m}$ and $30 \mathrm{~m} \times 30 \mathrm{~m}$. Four anchor nodes are respectively located at four corners and 1000 sensor nodes are randomly placed in the region. We assume that $P L 0=-60 d B$ and the zero-mean Gaussian noise $N_{\alpha}$ in Eq. 1 has a standard deviation of $3 d B$. We obtain the measured distance $d_{i}$ by Eq. (1). We set the degree $g=1.6$ in WCL [3].

Fig. 2, Fig. 3, and Fig. 4 show the localization error versus the path loss exponent for different network sizes by using the four algorithms. It can be observed from these three figures that as the network size increases, the error in location estimation increases for all four localization algorithms. The explanation is that the error of the RSS measurement increases as the network size increases. For all four localization algorithms, the localization error does not change much as the path loss exponent increases. But the results show that the proposed two weighted centroid algorithms achieve a smaller localization error than WCL and AMWCL-RSSI. This is because the weights are reasonably assigned to each anchor node due to the normal distribution. WCL and AMWCL-RSSI have a larger localization error because of the influence of the RSS. It can also be seen that PND and PNS have the advantage of higher accuracy and little influence from environment.

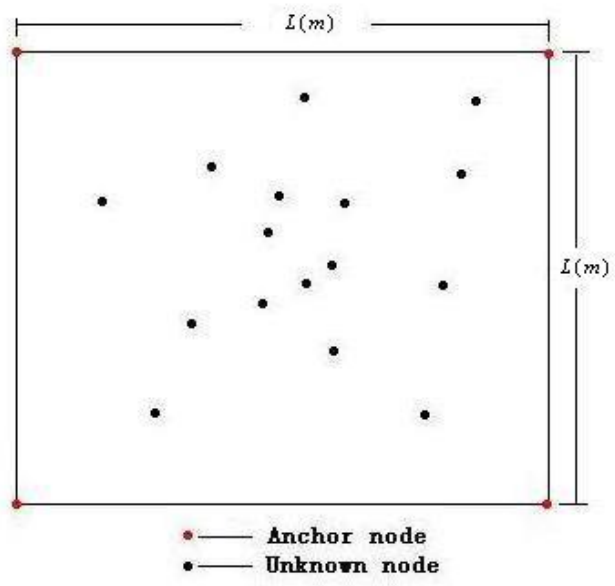

Figure 1. The network size as $\sigma^{2}$

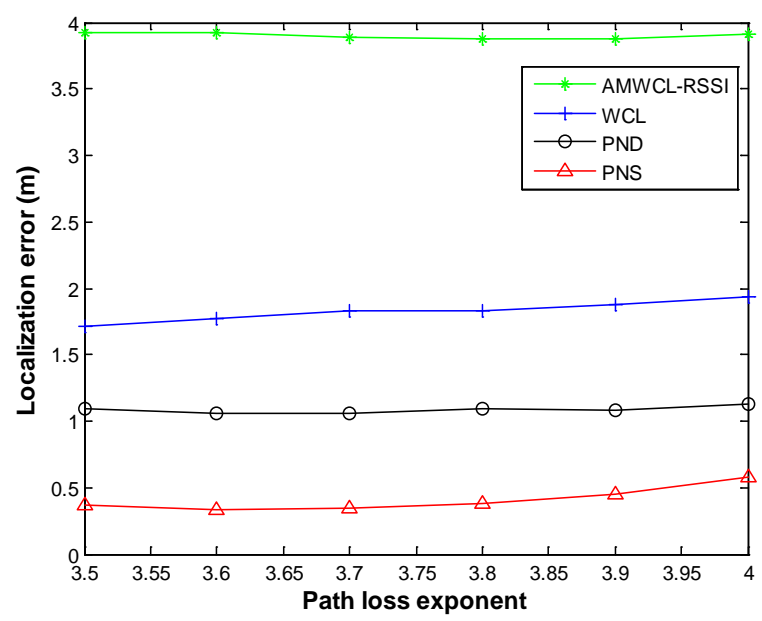

Figure 2. Network size $10 \mathrm{~m} \times 10 \mathrm{~m}$

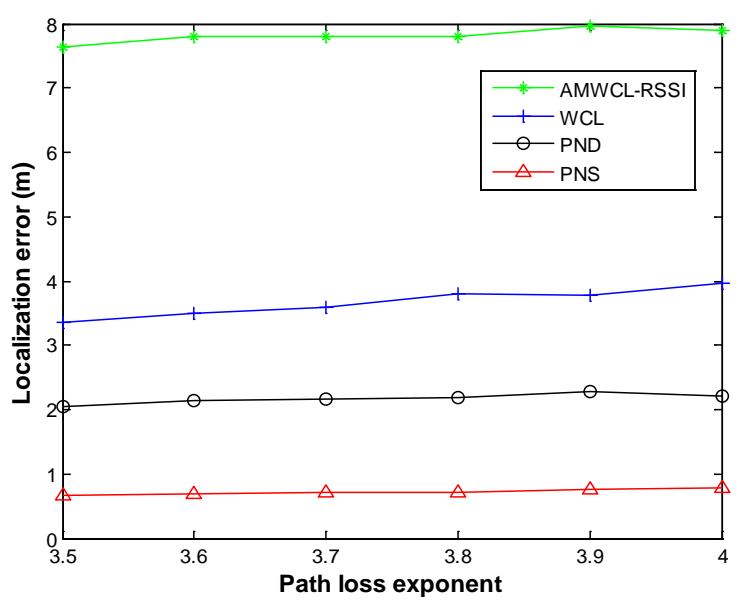

Figure 3. Network size $20 \mathrm{~m} \times 20 \mathrm{~m}$ 


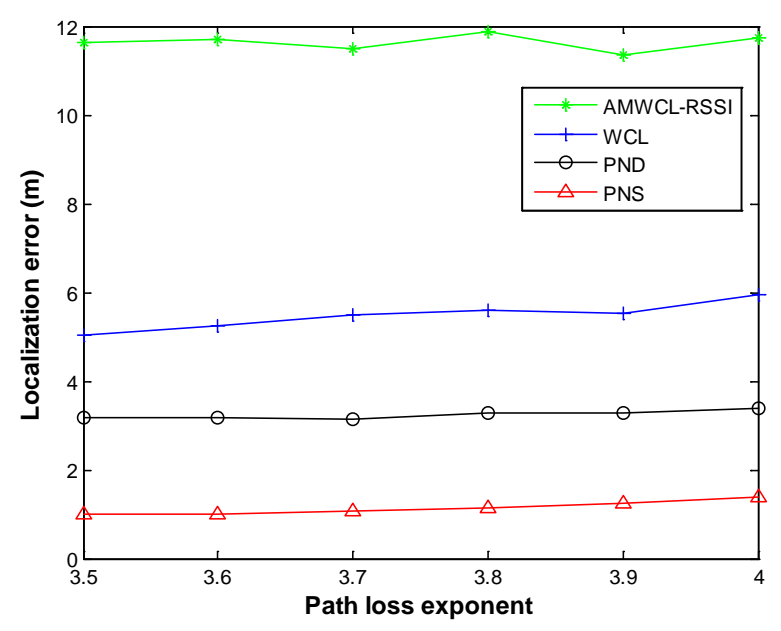

Figure 4. Network size $30 \mathrm{~m} \times 30 \mathrm{~m}$

TABLE I. AVERAgE LOCALIZATION ERRORS OF FOUR ALGORITHMS IN THREE NETWORK SIZES

\begin{tabular}{|c|c|c|c|}
\hline $\begin{array}{c}\text { Average } \\
\text { localization errors } \\
\text { (meters) }\end{array}$ & $10 \mathrm{~m} \times 10 \mathrm{~m}$ & $20 \mathrm{~m} \times 20 \mathrm{~m}$ & $30 \mathrm{~m} \times 30 \mathrm{~m}$ \\
\hline AMWCL-RSSI & 3.9 & 7.8 & 11.7 \\
\hline WCL & 1.71 & 3.3 & 5.1 \\
\hline PND & 1.05 & 2 & 3.25 \\
\hline PNS & 0.4 & 0.6 & 0.9 \\
\hline
\end{tabular}

Table 1 extracts the data from Fig. 1, 2, and 3 specifically for the path loss exponent $\beta=3.5$. Table 1 shows the average localization errors of four algorithms in three network sizes.

From Table 1, when network size is $10 \mathrm{~m} \times 10$, compared with WCL, PND can improve the localization accuracy by up to $38.5 \%$ and PNS can improve the localization accuracy by up to $76.6 \%$. Compared with AMWCL-RSSI, PND can improve the localization accuracy by up to $73 \%$ and PNS can improve the localization accuracy by up to $89.7 \%$. PNS outperforms PND is because PNS reduces the probability that $d_{i}$ is out of the range $(-3 \sigma,+3 \sigma)$.

From Table 1, when network size is $20 \mathrm{~m} \times 20$, compared with WCL, PND can improve the localization accuracy by up to $39.4 \%$ and PNS can improve the localization accuracy by up to $81.8 \%$. Compared with AMWCL-RSSI, PND can improve the localization accuracy by up to $74.3 \%$ and PNS can improve the localization accuracy by up to $92.3 \%$.

From Table 1, when network size is $30 \mathrm{~m} \times 30$, compared with WCL, compared with WCL, PND can improve the localization accuracy by up to $36.2 \%$ and PNS can improve the localization accuracy by up to $82.3 \%$. Compared with AMWCL-RSSI, PND can improve the localization accuracy by up to $72.2 \%$ and PNS can improve the localization accuracy by up to $92.3 \%$.

From Table 1, we can see that the localization error of the two proposed weighted centroid algorithms increases slowly as the network size increases. The reason is because the two proposed algorithms reduce the errors of the weights by using the normal distribution. The weight evenly changes with the measured distance according to the normal distribution. However, the localization error of WCL and AMWCL-RSSI increase quickly is because WCL and AMWCL-RSSI cannot better overcome the negative effect such as the error of the RSS measurements and an increase in the network size

\section{B. Real Experiment}

In this section, we test the performance of the studied localization schemes through a real experiment. A wireless node is based on a MSP430 microprocessor and equipped with a compliant Chipcon CC2500 wireless module. In the experiments, we tested three network sizes $10 \mathrm{~m} \times 10 \mathrm{~m}$, $20 \mathrm{~m} \times 20 \mathrm{~m}$ and $30 \mathrm{~m} \times 30 \mathrm{~m}$ in Anhui University's campus lawn. Four anchors are respectively placed in four corners. In order to obtain the average localization error of the whole region, 25 sensor nodes are uniformly deployed in the experimental region, corresponding to the deployment map in Fig. 5. We obtain the parameters in Eq. 1 through the RSS measurements. The experiment results are listed in Table 2.

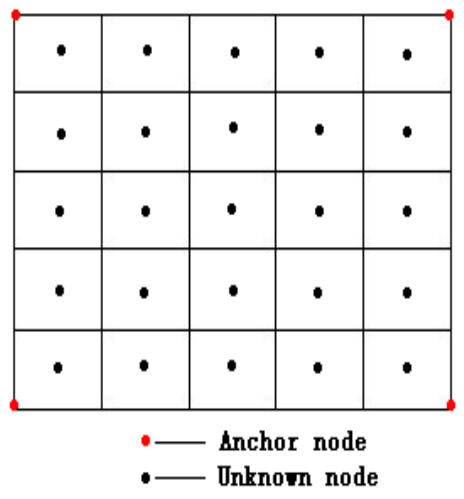

Figure 5. The deployment map of nodes

TABLE II. AVERAGE LOCALIZATION ERRORS

\begin{tabular}{|c|c|c|}
\hline network size & PND & PNS \\
\hline $10 \mathrm{~m} \times 10 \mathrm{~m}$ & $1.22(\mathrm{~m})$ & $0.46(\mathrm{~m})$ \\
\hline $20 \mathrm{~m} \times 20 \mathrm{~m}$ & $2.34(\mathrm{~m})$ & $0.78(\mathrm{~m})$ \\
\hline $30 \mathrm{~m} \times 30 \mathrm{~m}$ & $3.28(\mathrm{~m})$ & $1.05(\mathrm{~m})$ \\
\hline
\end{tabular}

Table 2 shows the average localization error of the two proposed localization algorithms in three network sizes. In Table 2, it can be observed that as the network size increases, the average localization error increases for the two proposed localization algorithms. Compared with the average localization error of PND and PNS in TABLE 1, the average localization error of PND and PNS in Table 2 is slightly bigger. From Table 2, we clearly know that the average localization errors of PNS in three network sizes are about $0.46 \mathrm{~m}, 0.78 \mathrm{~m}$ and $1.05 \mathrm{~m}$, respectively. 


\section{CONCLUSIONS}

In this paper, our work in weighted centroid localization is mainly used for planned deployment of anchor nodes in WSNs. Many weighted centroid localization schemes have been widely used for WSNs. the errors of these schemes are usually high in practical deployments. Moreover, too many anchor nodes will increase the cost, and complex environment will also increase the positioning error. To solve these problems, we have proposed two weighted centroid localization algorithms, namely PND and PNS, which can use normal distribution to improve the localization accuracy. The normal distribution reflects the randomness of the nodes distribution, and the relationship between the weight and the measured distance. More importantly, the variance can be set by the network size because presetting the network size can reduce the probability that $d_{i}$ is out of the range $(-3 \sigma,+3 \sigma)$. For PND, anchor nodes can be randomly deployed in the network. But, for PNS, we must preset the network size, and anchor nodes must be deployed at the edge of the selected area. Our experimental and simulation results demonstrated that the two proposed weighted centroid localization algorithms have a small localization error. It also has been shown that PND and PNS have the advantage of higher accuracy and little influence from environment. Hence, the proposed two algorithms can be applied to the occasion of higher accuracy and little influence from environment. Because PNS needs preset the network size, so we plan to solve the disadvantage in the future work.

\section{ACKNOWLEDGEMENT}

This work was supported in part by the Anhui Province education department general project (NO. AQKJ2015B005)

\section{REFERENCES}

[1] J.F. Huang, G.Y. Chang, G.H. Chen, A Historical-Beacon-Aided Localization Algorithm for Mobile Sensor Networks, submitted to: IEEE Transactions on Mobile Computing (2015).

[2] Lasla. N., Younis. M.F., Ouadjaout. A., Badache. N, An Effective Area-Based Localization Algorithm for Wireless Networks, submitted to: IEEE Transactions on Computers (2015).

[3] J. Blumenthal, R. Grossmann, F. Golatowski, D. Timmermann, Weighted Centroid Localization in Zigbee-based Sensor Networks, submitted to: IEEE International Symposium on Intelligent Signal Processing (2007).

[4] Hongyu Shi, A new weighted centroid localization algorithm based on RSSI, submitted to: International Conference on Information and Automation (ICIA) (2012).

[5] M.S. Jiang, S.Z. Lu, Low Velocity Impact Localization on CFRP Based on FBG Sensors and ELM Algorithm, submitted to: IEEE Sensors Journal (2015).

[6] Jun Wang , Urriza, P., Yuxing Han, Cabric, D., Weighted Centroid Localization Algorithm: Theoretical Analysis and Distributed Implementation, submitted to: IEEE Transactions on Wireless Communications ( 2011)

[7] J. J. Robles, J. S. Pola, and R. Lehnert, Extended Min-Max algorithm for position estimation in sensor networks, submitted to: 2012 9th Workshop on Positioning Navigation and Communication (2012).

[8] Bandiera. F., Coluccia. A., Ricci. G., A Cognitive Algorithm for Received Signal Strength Based Localization, submitted to: IEEE Transactions on Signal Processing (2015).

[9] ShihHau F., TsungNan L., Cooperative multi-radio localization in heterogeneous wireless networks, submitted to: IEEE Transactions on Wireless Communications (2010). 